\title{
A percepção das puérperas aos cuidados prestados pela equipe de enfermagem quanto ao seu puerpério
}

\author{
The perception of puerpers to the care provided by the nursing team regarding their puerperium
}

La percepción de los puerpers a la atención prestada por el equipo de enfermería respecto a su

puerpério

\section{Resumo}

Introdução: O período puerperal constitui-se em um momento de fragilidade, demandando dos profissionais de saúde uma atenção qualificada e holística, focada no contexto sociocultural, familiar e na avaliação dos cuidados ao trinômio mãe-criança-família. Objetivo: Analisar a percepção da puérpera aos cuidados prestados por equipe de Estratégia Saúde da Família quanto ao seu puerpério em uma cidade do interior do Maranhão. Metodologia: Esse estudo é do tipo exploratório, com uma abordagem quanti-qualitativa. A coleta de dados aconteceu no período de agosto de 2021. Resultados: Observou-se que existe uma lacuna no conhecimento das puérperas em quanto ao seu puerpério pela equipe de saúde como todo, notando-se que o enfoque dos profissionais de saúde é no recém-nascido, esquecendo-se da mulher em seu estado biopsicossocial. Conclusões: Conclui-se que a relação entre o profissional enfermeiro e as puérperas está baseada em três pilares, capacidade resolutiva, aptidão para o ensino e contato humanizado, sendo estes fundamentais para uma melhor promoção e prevenção de saúde.

Palavras-chave: Período pós-parto; Enfermagem; Saúde da mulher.

\begin{abstract}
Introduction: The puerperal period constitutes a moment of fragility, demanding qualified and holistic care from health professionals, focused on the sociocultural and family context and on the evaluation of mother-child-family care. Objective: To analyze the perception of the puerpery woman to the care provided by a Family Health Strategy team regarding her puerperium in a city in the interior of Maranhão. Methodology: This study is exploratory, with a quantitative-qualitative approach. Data collection took place in august 2021. Results: It was observed that there is a gap in the knowledge of the puerperal women regarding their puerperium by the health team as a whole, noting that the focus of health professionals is on the newborn, forgetting the woman in her biopsychosocial state. Conclusions: It is concluded that the relationship between the nursing professional and the puerpery women is based on three pillars, problem-solutive capacity, aptitude for teaching and humanized contact, which are fundamental for better health promotion and prevention.
\end{abstract}

Keywords: Postpartum period; Nursing; Women's health. 


\section{Resumen}

Introducción: El período puerperal constituye un momento de fragilidad, demandando una atención cualificada y holística por parte de los profesionales de la salud, centrada en el contexto sociocultural y familiar y en la evaluación de la atención materno-infantil-familiar. Objetivo: Analizar la percepción de la mujer puerpery a la atención prestada por un equipo de Estrategia de Salud de la Familia con respecto a su puerperio en una ciudad del interior de Maranhão. Metodología: Este estudio es exploratorio, con un enfoque cuantitativo-cualitativo. La recolección de datos tuvo lugar en agosto de 2021. Resultados: Se observó que existe una brecha en el conocimiento de las puerperales respecto a su puerperio por parte del equipo de salud en su conjunto, observando que el foco de los profesionales de la salud está en el recién nacido, olvidando a la mujer en su estado biopsicosocial. Conclusiones: Se concluye que la relación entre el profesional de enfermería y las mujeres puerpérpicas se basa en tres pilares, la capacidad solutiva-problema, la aptitud para la enseñanza y el contacto humanizado, que son fundamentales para una mejor promoción y prevención de la salud.

Palabras clave: Período post parto; Enfermería; Salud de la mujer.

\section{Introdução}

O pós-parto ou puerpério é um período caracterizado pela fase ativa do ciclo gravídico-puerperal, refletido por mudanças biopsicossociais que começam após a expulsão do feto e do conteúdo do útero gravídico, sendo marcado por seis semanas, tendo três períodos distintos como o puerpério imediato, tardio e remoto. Esse é o momento que corpo está retornando a sua normalidade e se readaptando após as alterações da gravidez e parto (Laporte, Zangirolani \& Medeiros, 2020).

O período puerperal constitui-se em um momento de fragilidade, demandando dos profissionais de saúde uma atenção qualificada e holística, focada no contexto sociocultural, familiar e na avaliação dos cuidados ao trinômio mãe-criança-família (Garcia et al., 2021) (Laporte, Zangirolani \& Medeiros, 2020).

A mulher em seu puerpério passa por transformações ambivalentes desde o parto ao cuidado com recém-nascido, podendo ser de alivio a medo, desconforto físico a ansiedade de amamentar, decepção a autoconfiança, definido assim que as vivencias puerperais são a parte do processo de adaptação do corpo em sua totalidade a nova vida (Navas Arrebola et al., 2021) (Brasil, 2006).

Com essa transição de etapas, estabeleceram-se os objetivos que se atendem ao estado geral da mulher e do recémnascido, como apoio ao aleitamento materno, orientação ao planejamento familiar, identificação de situações de riscos e a integração da relação binômino mãe-filho, assim garantindo cuidada a saúde de forma integral a ambos (Laporte, Zangirolani \& Medeiros, 2020).

Essa a assistência a puérpera inicia-se no ambiente hospitalar, determinada pela fase imediata de 2-4 horas após a expulsão, ocorrendo às primeiras alterações fisiológicas e psicológicas deste novo ciclo. Os cuidados administrados neste momento são importantes para definição da alta hospitalar e sendo continuada pela equipe de Estratégia de Saúde da Família (ESF), sendo pautadas no puerpério tardio e remoto seguido até as seis semanas estabelecidas como consultas e visitas domiciliares puerperais, puericultura e o planejamento familiar (Silva et al., 2020).

A equipe de Estratégia Saúde da Família é a parte fundamental para que aconteça um puerpério saudável destas mulheres, sendo o enfermeiro o organizador dos cuidados, pautando-se em promover ações que mulher se recupere principalmente nas visitas domiciliares, devendo conhecer a realidade de cada. Essas condutas são orientações para aleitamento, atenção ao recém-nascido e o planejamento familiar para espaçar nova gravidez (Silva et al., 2020).

Por fim, a relevância da pesquisa está pautada na reflexão da puérpera sobre os cuidados no puerpério, por atrelar a vivência destas mulheres para melhor atendimento e efetivação das políticas voltadas a esse público, conhecendo as singularidades impostas por cada realidade. Sendo a pergunta norteadora: qual a percepção da puérpera aos cuidados prestados pela equipe de enfermagem quanto ao seu puerpério em uma cidade do interior do Maranhão?

Com objetivo geral de analisar a percepção da puérpera aos cuidados prestados pela equipe de enfermagem quanto ao seu puerpério em uma cidade do interior do Maranhão. 


\section{Metodologia}

A presente pesquisa iniciou-se após a aprovação do Comitê de Ética em Pesquisa da Universidade Ceuma (CEP/UNICEUMA). Respeitando todos os preceitos éticos e legais de acordo com a Resolução $n^{\circ}$ 466/12, que regulamenta a pesquisa com seres humanos, parecer 4.874.436.

Esse estudo é do tipo exploratório, com uma abordagem quanti-qualitativa, conforme o modelo Standards for Quality Improvement Reporting Excellence 2.0 (SQUIRE), com intuito de avaliar as intervenções utilizadas pela equipe de enfermagem (Lakatos \& Marconi, 2017). A pesquisa foi desenvolvida em 3 Unidades Básicas de Saúde (UBS) na cidade de Campestre, no Estado do Maranhão, composta por 1 equipe em cada Unidade com médico, enfermeiro, técnico de enfermagem e agente comunitário de saúde na Estratégia Saúde da Família. As ações realizadas são de acompanhamento de pré-natal de baixo risco, puericultura, HIPERDIA (hipertensos e diabéticos), imunização e preventivo de câncer de colo de útero e mama, além de promoção e prevenção a saúde em um contexto multidisciplinar a população.

As participantes do estudo foram puérperas atendidas pelas 3 equipes com parto entre junho, julho e agosto de 2021.

Os critérios de inclusão estabelecidos para presente pesquisa foram de puérperas dentre os 45 dias (6 semanas) preconizados, idade estabelecida de 18-40 anos, justificando-se como a idade reprodutiva adequada (Brasil, 2012), primíparas e multíparas e estão sendo acompanhadas no puerpério pela Estratégia de Saúde da Família local.

Os critérios de exclusão compuseram puérperas que perderam recém-nascido, que tiverem parto de fevereiro a maio, menores de idade entre 14 a 17 anos e que não concordaram com Termo de Consentimento Livre e Esclarecido.

A coleta de dados aconteceu no período de agosto de 2021 por meio de questionário estruturado para obter os dados esperados, sendo coletados por encaminhamento via e-mail com link ao acesso Google Formulários.

Diante o cenário da SARS-CoV-2 (pandemia do COVID-19) teve-se que reestruturar as pesquisas, com isso, o método adaptado para coletar os dados foi de forma online, assim não colocando as puérperas e sua família em risco (Brito et al., 2020) ocorrendo o estudo de forma segura e sem implicações de contágio do COVID-19.

As pesquisadoras do presente estudo levantaram os registros das Unidades Básicas de Saúde com os agentes comunitários de saúde de cada bairro, identificando-se 40 contatos elegíveis, consecutivamente por meio do aplicativo de mensagens Whatsapp ocorreu o contato das pesquisadoras com as participantes certificando-se que os critérios de inclusão e exclusão seriam seguidos, esclarecendo dúvidas sobre Termo do Consentimento Livre e Esclarecido.

Com o interesse de participar, encaminhou-se um e-mail as participantes com convite, elucidadas dos objetivos, além do link do questionário e Termo do Consentimento Livre e Esclarecido assinado para que as participantes pudessem ler e consentir de forma virtual e arquiva-lo.

Os dados foram organizados de acordo com questionário elaborado que visou identificar as condições sociais e pessoais da puérpera, além de analisar a atuação da Estratégia de Saúde da Família nos cuidados ao puerpério, o atendimento puerperal e conhecimento sobre as condições de saúde como autocuidado, percepções ao puerpério, relacionamento afetivo e sexualidade. Além de serem analisados por estatística descritiva simples que visa um resumo sobre amostras observadas que podem ser quantitativas ou visualmente por meio de tabelas e quadros, sendo a descrição das informações obtidas (Rodrigues, Lima \& Timbó, 2017).

A amostra do estudo não foi intencional, sendo obtidos por identificação das puérperas que foram elegíveis por meio dos critérios de inclusão e exclusão, com amostra final de 17 participantes elencadas detalhadas na Figura 1: 
Figura 1 - Procedimentos utilizados para amostra da pesquisa, Campestre, Maranhão, Brasil, 2021.

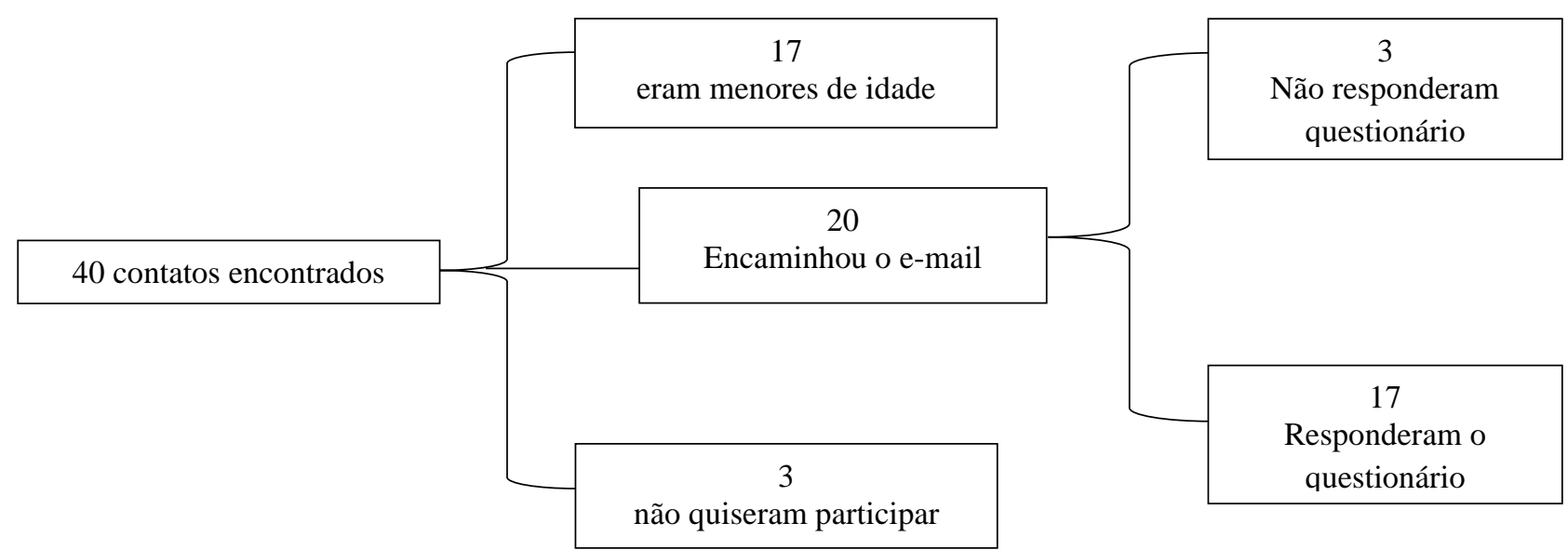

Fonte: Autores (2021).

A coleta de dados aconteceu no período de agosto de 2021 por meio de questionário estruturado para obter os dados esperados, sendo coletados por encaminhamento via e-mail com link ao acesso Google Formulários.

Diante o cenário da SARS-CoV-2 (pandemia do COVID-19) teve-se que reestruturar as pesquisas, com isso, o método adaptado para coletar os dados foi de forma online, assim não colocando as puérperas e sua família em risco (Brito et al., 2020) ocorrendo o estudo de forma segura e sem implicações de contágio do COVID-19.

As pesquisadoras do presente estudo levantaram os registros das Unidades Básicas de Saúde com os agentes comunitários de saúde de cada bairro, identificando-se 40 contatos elegíveis, consecutivamente por meio do aplicativo de mensagens Whatsapp ocorreu o contato das pesquisadoras com as participantes certificando-se que os critérios de inclusão e exclusão seriam seguidos, esclarecendo dúvidas sobre Termo do Consentimento Livre e Esclarecido.

Com o interesse de participar, encaminhou-se um e-mail as participantes com convite, elucidadas dos objetivos, além do link do questionário e Termo do Consentimento Livre e Esclarecido assinado para que as participantes pudessem ler e consentir de forma virtual e arquiva-lo.

Os dados foram organizados de acordo com questionário elaborado que visou identificar as condições sociais e pessoais da puérpera, além de analisar a atuação da Estratégia de Saúde da Família nos cuidados ao puerpério, o atendimento puerperal e conhecimento sobre as condições de saúde como autocuidado, percepções ao puerpério, relacionamento afetivo e sexualidade. Além de serem analisados por estatística descritiva simples que visa um resumo sobre amostras observadas que podem ser quantitativas ou visualmente por meio de tabelas e quadros, sendo a descrição das informações obtidas (Rodrigues, Lima \& Timbó, 2017).

As pesquisas foram completadas com artigos de 2016 a 2021 na Base de Dados Scientific Electronic Library Online (SCIELO) e Literatura Latino-Americana e do Caribe em Ciências da Saúde (LILACS), acerca dos descritores em saúde (DECS) Período Pós-Parto e Enfermagem.

\section{Resultados}

As 17 puérperas participantes da pesquisa possuem idade média diversificada, em sua maioria 22 anos, sendo a idade mínima encontrada de 18 anos e máxima de 34 anos. O estado civil apresentou-se em 35,3\% em união estável, 11,8\% casadas e 52,9\% solteiras. A escolaridade baseou-se ensino fundamental completo 5,9\%, ensino fundamental incompleto 5,9\%, ensino médio incompleto em $35,3 \%$, ensino médio completo $41,2 \%$ e ensino superior completo $11,7 \%$. A ocupação das puérperas em 
do $\operatorname{lar} 88,2 \%$ e trabalham fora $11,8 \%$. A renda mensal caracterizou-se de $80 \%$ salário mínimo e $13,3 \%$ mais de 2 salários mínimos e somente $6,7 \%$ mais de 3 salários mínimos. A ocupação do companheiro em $60 \%$ em não tenho companheiro e $40 \%$ em empregados em lojas comercias.

Sobre histórico de gestação anterior, afirmaram baixo risco em 82,4\% e alto risco com histórico de aborto, gemelaridade, natimorto, eclampsia ou diabetes gestacional em 17,6\%. Já sobre comparecimento as consultas de pré-natais, os resultados foram satisfatórios com $88,2 \%$ de presença aos três trimestres do pré-natal contra 11,8\% não compareceu fielmente. Já número de consultas $52,9 \%$ foi a mais de 6 consultas contra 35,3\% que foram de 4 a 6 e 11,8\% de 2 a 4 .

Já ao conhecimento sobre seu puerpério, houve-se similaridade das respostas conforme a Tabela 1:

Tabela 1 - O conhecimento das puérperas quanto ao puerpério, Campestre, Maranhão, Brasil, 2021

\begin{tabular}{cc}
\hline Conhecimento das puérperas & Resultado \\
\hline $\begin{array}{c}\text { Sei sobre o que familiares falam como } \\
\text { sangramento é comum, dor ao amamentar e a } \\
\text { diminuição de desejo sexual. }\end{array}$ & $70,6 \%$ \\
\hline $\begin{array}{c}\text { Sei bem pouco sobre o que é e quais os cuidados } \\
\text { devo ter }\end{array}$ & $29,4 \%$ \\
\hline
\end{tabular}

Fonte: Autores (2021).

Relacionado às orientações recebidas na maternidade, às mulheres relataram que foram dadas orientações gerais em quanto ao bebê $52,9 \%$ contra $23,5 \%$ que receberem informações imprecisas e as que não receberam orientação nenhuma sendo de $23,6 \%$.

Contudo, teve-se grande diversidade de respostas quanto às consultas puerperais. Em relação à primeira consulta $70,6 \%$ recebeu a visita e a $29,4 \%$ que não recebeu nenhuma durante todo puerpério.

Sobre planejamento familiar pós-puerperal e uso de métodos contraceptivos, as puérperas relataram em sua maioria estarem desgostosas com essas informações, como mostra a Tabela 2:

Tabela 2 - Informações fornecidas sobre planejamento familiar pós-puerperal, Campestre, Maranhão, Brasil, 2021.

\begin{tabular}{c|c}
\hline Informações fornecidas & Resultado \\
\hline $\begin{array}{c}\text { Nunca recebi nenhuma informação de planejamento } \\
\text { familiar e nem foi me informado sobre uso de } \\
\text { anticontraceptivos. }\end{array}$ & $82,4 \%$ \\
\hline $\begin{array}{c}\text { Recebi informações por terceiros, como meus familiares } \\
\text { sobre uso de anticoncepcional no pós-parto. }\end{array}$ & $8.8 \%$ \\
\hline $\begin{array}{c}\text { Recebi informações da enfermeira na consulta puerperal } \\
\text { dentre os primeiros 7 dias sobre planejamento familiar e } \\
\text { uso de contraceptivos }\end{array}$ & $8,8 \%$ \\
\hline
\end{tabular}

Fonte: Autores (2021).

A maioria está sendo acompanhada por enfermeiro, médico e agente comunitário de saúde em 52,9\% e os outros $47,1 \%$ pelo enfermeiro e agente comunitário de saúde.

Destaca-se a satisfação das usuárias das Unidades Básica de Saúde como sua rede de apoio e prestadora de cuidados do puerpério, elencado a equipe de enfermagem, seguido à Tabela 3: 
Tabela 3 - Satisfação das puérperas ao atendimento da equipe de enfermagem, Campestre, Maranhão, Brasil, 2021.

\begin{tabular}{l|c}
\hline \multicolumn{1}{c|}{ Satisfação } & Resultado \\
\hline $\begin{array}{l}\text { A equipe de enfermagem da Estratégia Saúde da Família forneceu informações do } \\
\text { meu "resguardo" como sangramento, vida sexual, métodos contraceptivos, } \\
\text { cuidados ao amamentar, higiene, alimentação e ingesta hídrica. }\end{array}$ & $45,3 \%$ \\
\hline $\begin{array}{l}\text { A equipe de enfermagem da Estratégia Saúde da Família não forneceu informações } \\
\text { do meu "resguardo" como sangramento, vida sexual, métodos contraceptivos, } \\
\text { cuidados ao amamentar, higiene, alimentação e ingesta hídrica. }\end{array}$ & $27,35 \%$ \\
\hline $\begin{array}{l}\text { A equipe de enfermagem da Estratégia Saúde da Família forneceu poucas } \\
\text { informações do meu "resguardo" como sangramento, vida sexual, métodos } \\
\text { contraceptivos, cuidados ao amamentar, higiene, alimentação e ingesta hídrica. }\end{array}$ & $27,35 \%$ \\
\hline
\end{tabular}

Fonte: Autores (2021).

Apesar desses percalços, as puérperas em sua grande maioria afirmaram em 52,5\% a procura em primeiro lugar com equipe de estratégia saúde da família contra 47,5\% que recorrem à maternidade ou hospital público.

Por fim, destaca-se que a concepção das puérperas afim de seu puerpério, mostrando que 68,8\% estão com boas vivencia no puerpério, contra $18,8 \%$ que estão com dificuldades e $12,4 \%$ que sente que precisa de mais informações.

\section{Discussão}

A caracterização da idade e do estado civil no puerpério se tornam importantes para definir o estado puerperal de forma mais objetiva, como mostra a pesquisa Campos PA \& Féres-Carneiro (2021).

puérperas com idade de 25 a 30 anos apresentaram vivências pós-parto mais tranquilo, onde se observou que mulheres que obtiveram auxílio de seus companheiros e família, além de relatarem que se prepararam de forma mais adequada desde a gestação. Outra pesquisa complementa que quanto maior grau de escolaridade, mais informações são buscadas pelas puérperas, principalmente quanto aos cuidados que se devem ser seguidos tanto com seu puerpério como com recém-nascido, assim observa-se que no estudo atual a idade manifestou-se como fator preponderante ao nível de saberes sobre estado puerperal. (Figueiredo et al., 2018),

Além disto, o Ministério da Saúde (2012) preconiza no mínimo 6 consultas de pré-natal intercalado entre enfermeiro e médico em gestação de baixo risco' com isso, esses dados se apresentaram de forma satisfatória, sendo uma porcentagem mínima que não compareceu as 6 consultas, denotando que as orientações da gestação ao puerpério possam ser passadas de forma correta, construindo um pós-parto saudável.

Entretanto, notou-se que há precariedade nestas informações, sendo a insatisfação dessas puérperas válidas. É indicado que a partir das 35 semanas que a equipe de saúde ministre os cuidados ao estado puerperal, principalmente sobre imediato, tardio e remoto. Como no puerpério imediato que se inicia no $1^{\circ}$ ao $10^{\circ}$ dia e necessita-se que a puérpera identifique os sinais esperados como sangramento (loquios), cólicas pélvicas, alterações de humor, sinais de risco de uma possível depressão pós-parto, complicações da amamentação com ingurgitamento mamário, atenção com a pele, alimentação e ingesta hídrica. O do tardio, que começa a partir do $11^{\circ}$ a $42^{\circ}$ dia, onde pode acontecer um atraso menstrual e dores abdominais frequentemente pela involução uterina e dos órgãos ao seu local habitual e por último do puerpério remoto a partir do $43^{\circ}$ dia, que marca retorno da vida sexual da mulher e o planejamento familiar para alongar uma nova gestação (Baratieri \& Natal, 2019) (Nagila et al., 2019).

A carência desses conhecimentos provoca ainda mais intercorrências puerperais, sendo uma transição dolorosa. Nesta perspectiva, destaca-se não só a importância das Unidades Básicas de Saúde, mas como da maternidade em trazer esses dados. 
Neste discurso, observa-se que são os profissionais da maternidade que não se atentam também a essa situação, sendo explicado pelo período de internação ser curto variando de 24 horas para o parto normal e 48 horas para o parto cesáreo. Com isso, os profissionais de saúde acabam postergar as informações importantes aos puerpérios, limitando-se a amamentação, cuidado com as mamas e primeiro contato com recém-nascido, se cesáreo, outros cuidados pertinentes a cirurgia (Silva, Russo \& Nucci, 2021).

Assim, ainda destaca-se que se observou que o serviço de saúde delimita-se ao recém-nascido, focado na recuperação da puérpera em sua visão fisiológica, esquecendo-se do bem estar tanto social como psicológico dessa mulher, onde ela deve administrar atenção não somente à criança, mas também a si mesma, de maneira que se lembre-se de que mesmo após o nascimento do filho, existe um corpo que necessita de cautela com alimentação e ingesta hídrica igualmente como precisa de descanso e tempo consigo mesma, destacando é importante que todos os membros da familiar participem deste processo e auxiliem a puérpera a enxergar-se como um ser humano que precisa de cuidados (Garcia et al., 2021) (Boing et al., 2021).

Ainda neste foco, notou-se o planejamento familiar é escasso, ocorrendo que as mães em sua maioria não sabem significado e nem seu objetivo, devendo existir a orientação desde maternidade a Atenção Primária, visto que aleitamento materno é impreciso se não for implementado durante os 6 primeiros meses, assim destaca-se que vida sexual desta mulheres deve ser um foco do puerpério, como cita Ministério da Saúde, o planejamento deve ser realizado durante gestação e pós parto, preconizado orientação ao retorno da sexualidade e da contracepção (Dodou et al., 2017) (Paris, Montigny\& Pelloso, 2021).

Ao fazer a escolha contraceptiva no período puerperal, além das características inerentes a qualquer método, tais como eficácia, segurança, eventos adversos e reversibilidade, deve-se atentar para a possibilidade de efeitos sobre a lactação e o recém-nascido. Algumas opções podem ser limitadas devido às alterações que podem causar na qualidade ou na quantidade do leite. Os métodos devem ser avaliados de acordo com os critérios de elegibilidade para uso de contraceptivos (Correa et al., 2017).

O intervalo intergestacional para mais de 2 anos é vantajoso a relação materno-fetal, principalmente entre 3 a 4 anos de parto anterior, sendo uma taxa significativa para sobrevivência e melhor qualidade de vida destas crianças, já intervalos curtos estão associados parto prematuro, anemia e baixo peso ao nascer, sendo parte das diretrizes do planejamento familiar estas informações (Justino et al., 2021) (Paris, Montigny \& Pelloso, 2021).

Notou-se esse enfoque na criança, dificulta-se que as mulheres possam se sentir seguras no seu puerpério, principalmente sobre seus sentimentos que se torna em intensos durante esse ciclo. Ainda em atenção à saúde, percebeu-se que o enfermeiro e agente comunitário de saúde são os principais profissionais que as puérperas têm contato, sendo uma responsabilidade compartilhada que deveria integrar médico, odontólogo e enfermeiro, entretanto, é visto que este protocolo preconizado pelo Ministério da Saúde não é levado a sério, assim trazendo lacunas a equipe Estratégia Saúde da Família (Silva; Russo \& Nucci, 2021).

A Equipe Estratégia Saúde da família deve ser integrativa do cuidado à saúde, devendo- se leva as consultas domiciliares e os conhecimentos necessários. Observaram-se dois cenários, onde se houve as consultas puerperais em sua maioria, entretanto, as orientações não foram repassadas de acordo, diminuindo o vínculo com as puérperas (Paris, Montigny \& Pelloso, 2021).

Um estudo que se baseia no aspecto familiaridade na saúde denotou que quanto maior vinculo equipe e paciente, mais as ações são efetivas, além de que puérpera comparece mais a unidade, segue as orientações corretas e contribui mais com os profissionais de saúde. Por isso, a equipe necessita prestar os métodos necessários, principalmente a enfermagem que constitui como o elo neste meio, notou-se que quanto mais o enfermeiro é presente, mas as puérperas veem atenção básica como primeiro acesso (Silva et al., 2016). 
Assim, o enfermeiro torna-se o principal atendente as necessidades tanto físicas como psicossociais da mulher neste período, é ele que deve administrar os conhecimentos como puerpério, vivências com os familiares e o novo membro da família, assim como planejamento familiar e retorno da vida sexual e social. É ele que deve se atentar a intercorrências no puerpério, além que este momento de sentimentos opostos como de felicidade a dor, além das outras doenças que podem comprometer a saúde da mãe. Os cuidados no APS compreendem a emergência de se olhar de forma singular.

Notando-se que a enfermagem é o primeiro contato destas mães e a conexão do cuidado, refere-se que existe uma ambivalência na atenção encontrada, onde em sua maioria se sentem satisfeitas com atenção, porém, apresentam com lacunas que necessita serem melhoradas, principalmente para promoção e educação em saúde dessas mulheres terem um puerpério de qualidade

\section{Conclusões}

Diante do exposto, conclui-se que a relação entre o profissional enfermeiro e as puérperas está baseada em três pilares, capacidade resolutiva, aptidão para o ensino e contato humanizado, sendo estes fundamentais para uma melhor promoção e prevenção de saúde, tendo em vista que através do contato durante as consultas o enfermeiro tem a oportunidade de identificar os sinais e sintomas de situações de risco, orientar as puérperas e tomar decisões efetivas no processo do cuidar.

Além de que se é nítido que as dificuldades encontradas aos cuidados do puerpério são referentes às queixas do déficit em informações, sendo um problema da equipe em sua totalidade, necessitando que estas puérperas sejam assistidas de uma forma melhorada.

Também se observou que foram características pessoais diversas de cada puérpera, entretanto houve-se um choque de similaridade entre os saberes do seria os cuidados com puerpério, evidenciou-se que os dados acerca sobre esse tema foram adquiridos principalmente dos familiares, mostrando a insatisfação destas mulheres em relação ao atendimento em saúde, exibido que equipe necessita de mais ações em saúde focado em cuidados singulares ao puerpério, planejamento familiar, vida sexual e social.

Portanto, fica claro que o profissional enfermeiro precisa estar devidamente capacitado para atender às expectativas da parturiente, principalmente emocionais, visto que este é o momento de mudanças tanto fisiológicas como psicológicas neste novo ciclo

Como limitação da pesquisa, relata-se a restrição do número de participantes, principalmente pelo local de estudo ser uma unidade pequena, denotando assim dificuldades para conceber a amostra final do estudo.

Esse estudo contribui para enfermagem visando promoção de um atendimento especializado que leve em consideração à realidade da puérpera, desde os problemas socioeconômicos até os psicossociais, designado que o profissional de enfermagem possa se torna capaz de conquistar a confiança da paciente, melhorando a relação entre os dois e a partir daí, ampliando as possibilidades de intervenção, uma vez que com uma relação mais próxima e sólida, além de se capacitar de uma melhor forma, a fim de trazer os conhecimentos precisos a puérpera aos seus cuidados no puerpério.

É importante que trabalhos futuros se aprofundem nos cuidados a puérpera em ambiente hospitalar, visto que se identificou um déficit em artigos relacionados desse tema, de modo que o primeiro cuidado à mulher no período puerperal é dentro das maternidades, seguindo-se para as unidades básicas de saúde.

\section{Referências}

Laporte, A. S. C. M., Zangirolani, L. T. O., Medeiros, M. A. T. (2020). Prenatal and puerperium nutritional care from na integrality perspective in a city of Baixada Santista, São Paulo, Brazil. Rev. bras. saúde mater. infant. [Internet]. 20(1), 145-55.

Navas Arrebola, R. et al. (2021). Women's satisfaction with childbirth and postpartum care and associated variables. Rev Esc Enferm USP [Internet]. 55(1), 17 . 
Brito, S. B. P et al. (2020) Pandemia da COVID-19: o maior desafio do século xxi. Vigil. sanit. debate [Internet] 2020 [cited 202101 sep] 8 (2), $54-63$.

Baratieri, T \& Natal, S. (2019). Ações do programa de puerpério na atenção primária: uma revisão integrativa. Ciênc. Saúde Colet. [Internet]. 24(11), 42274238 .

Boing, A. F. et al. (2021). Métodos e aspectos operacionais de um estudo epidemiológico e de avaliação da Rede Cegonha. Revista Rev. bras. epidemiol [Internet]. 24(1), 1-13.

Brasil. (2013). Resolução ${ }^{\circ}$ 466, de 12 de dezembro de 2012. Dispõe sobre diretrizes e normas regulamentadoras de pesquisas envolvendo seres humanos. Diário Oficial [da] República Federativa do Brasil, Brasília.

Brasil. (2006). Manual técnico de pré-natal e puerpério. Brasília: Ministério da Saúde.

Campos, P. A., Féres-Carneiro, T. (2021). Sou mãe: e agora? Vivências do puerpério. Psicol. USP. [Internet]. 32(1), 1-9.

Corrêa, M. S. M. et al. (2017). Acolhimento no cuidado à saúde da mulher no puerpério. Cad. saúde pública. [Internet]. 2017.

Dodou, H. D. et al. (2017). Educational practices of nursing in the puerperium: social representations of puerperal mothers. Rev Bras Enferm [Internet]. 70(6): $1250-8$.

Figueiredo, J. V. et al. (2018). Pain in the immediate puerperium: nursing care contribution. Rev. bras. enferm. 71(3), 1343-1350.

Garcia, N. P. et al. (2021). The nursing process in postpartum consultations at Primary Health Care Units. Rev. Esc. Enferm. USP. [Internet]. 55(1), 1-8.

Justino, G. B. S. et al. (2021). Educação sexual e reprodutiva no puerpério: questões de gênero e atenção à saúde das mulheres no contexto da Atenção Primária à Saúde. Interface - Comunic., Saude, Educ. [Internet]. 25(1), 1-17.

Lakatos, E. M.; Marconi, M. A (2017). Metodologia científica. Atlas.

Nagila, N. L. F., Fabiane, F. D. A. G., Marcela, M. A. B., Jéssica, J. L. B., Mariana, M. C. M., Talyta, M. T. M. P., \& de Freitas Ranielder, R. F. (2019). Construção de um mapa de conversação para gestantes e puérperas sobre os cuidados com o recém-nascido. Revista Cubana de Enfermería, 35(2).

Paris, G. F., Montigny, F., Pelloso, S. M. (2021). Professional practice in caring for maternal grief in the face of stillbirth in two countries. Rev Bras Enferm [Internet]. 74(3), 1-7.

Quintão, V. C. et al. (2019). Reporting guidelines: tools to increase the completeness and transparency of your anesthesiology research paper. Rev. bras. anestesiol. [Internet]. 69(5). 429-431.

Rodrigues, C.F.S., Lima, F.J.C., Timbó, F.B. (2017). Importance of using basic statistics adequately in clinical research. Rev. bras. anestesiol. [Internet]. $67(6), 619-625$.

Silva, E. M. D., Marcolino, E., Ganassin, G. S., Santos, A. L. D., \& Marcon, S. S. (2016). Participação do companheiro nos cuidados do binômio mãe e filho: percepção de puérperas. Rev. pesqui. cuid. fundam.(Online), 8(1), 3991-4003.

Silva, F. L., Russo, J. e Nucci, M. (2021). Gravidez, parto e puerpério na pandemia: os múltiplos sentidos do risco. Horiz. antropol. [Internet]. 27(59), 245265.

Silva, L. P. da et al. (2020). Assistance to the puerperium and the construction of a flow chart for nursing consultation. Rev. bras. saúde mater. infant. [Internet].20(1), 101-113. 